\title{
Development of pH-Responsive Interpenetrating Polymer Networks of Polyacrylamide-g-Gum Arabica and Sodium Alginate for Gastroprotective Delivery of Gabapentin
}

\author{
S. PRAMANIK, J. DUTTAAND P. CHAKRABORTY* \\ Department of Pharmaceutics, Himalayan Pharmacy Institute, Majhitar, Sikkim 737136, India
}

Pramanik et al.: pH-Responsive Interpenetrating Polymer Networks of Polyacrylamide-g-Gum Arabica and Sodium Alginatee

\begin{abstract}
In the present work, a grafted polymer of polyacrylamide with gum arabica was synthesized as Gum acacia-g-polyacrylamide using microwave-assisted synthesis with ceric ammonium nitrate as a reaction initiator. The polymer was utilized in combination with sodium alginate to prepare interpenetrating polymer network hydrogel beads to effectively deliver gabapentin in gastrointestinal tract. The formulated interpenetrating polymer network microbeads showed $\mathrm{pH}$-responsive release in a different medium. The interpenetrating polymer network hydrogel beads were processed for different analytical characterization like Fourier transform infrared spectroscopy, differential scanning calorimetry, $\mathrm{X}$-ray powder diffraction and scanning electron microscopy. The Fourier transform infrared spectroscopy study confirms the formation of gum acacia-g-polyacrylamide whereas differential scanning calorimetry, $\mathrm{X}$-ray powder diffraction studies confirm the appearance of gabapentin in the interpenetrating polymer network microbead formulations. The release of the drug from the interpenetrating polymer network structured hydrogel beads was found higher in $\mathrm{pH} 6.8$ as compared to $\mathrm{pH} 1.2$ and $\mathrm{pH} 7.4$ indicating the effective delivery of gabapentin from the interpenetrating polymer network beads to upper duodenum for better absorption.
\end{abstract}

Key words: Interpenetrating polymer network, polyacrylamide, gum acacia-g-polyacrylamide, gabapentin, pH responsive

In recent years, polysaccharide polymers are extensively used as excipients in parenteral area, controlled drug release (CDR) and drug targeting to specific organs ${ }^{[1]}$. But natural polymer has some limitation in their reactivity. Hence, polymer mixture or blending are used for improving the physical properties, mechanical strength and poor biological performance of natural polymer ${ }^{[2]}$. An Interpenetrating Polymer Network (IPN) is the mixture of binary or more polymers which form a network like structure where one polymer is synthesized and/or cross-linked in the existence of another ${ }^{[3]}$. Each polymer network detains its individual properties so synergic action like strength or toughness is improved.

Gum arabic or Gum acacia (GA) is hardened shape natural gum which is collected from various species of the acacia tree. The source of GA is Acacia senegal and Vachellia (Acacia) seyal. GA is consisting of glycoproteins and polysaccharides. GA is the source of the sugar compound named arabinose and ribose. $\mathrm{GA}$ is soluble in water. The main chain consists of 1,3-linked $\beta$-D-galactopyranosyl units and the side chains are composed of two to five 1,3-linked $\beta$-Dgalactopyranosyl units which are joined to the main chain by 1,6 -linkages ${ }^{[4]}$. The galactose residues within the backbone chain similarly the ramified aspect chains such as arabinose, rhamnose and glucuronic acid, contain pendant hydroxyl groups $(-\mathrm{OH})$ which contain non-bonding electrons, easy to ionize using electron loving species or oxidizing agents such as ceric ions

This is an open access article distributed under the terms of the Creative Commons Attribution-NonCommercial-ShareAlike 3.0 License, which allows others to remix, tweak, and build upon the work non-commercially, as long as the author is credited and the new creations are licensed under the identical terms Indian J Pharm Sci 2021;83(3):473-482 
and hence create reactive sites for graft polymerization reactions to commence ${ }^{[5]}$.

Polyacrylamides (PAM) have been reported to be protein resistant and biocompatible ${ }^{[6]}$. It referred to as a PAM that is formed by the polymerization of acrylamide $\left(\mathrm{C}_{3} \mathrm{H}_{5} \mathrm{NO}\right)$, attained by the hydration of acrylonitrile. It has the unique property of being soluble in water that is hard glassy solid at room temperature. The polymer is hydrophilic and can form aqueous solutions of very high concentration ${ }^{[7]}$. It has a large number of pendant amide group on its backbone. Amide group has a high synthetic activity which can frame a progression of subordinates with numerous sorts of compounds. It is utilized in biomedical application.

Sodium alginate (SA) is a bioerodible natural polymer with linear chain structure of $(1 \rightarrow 4)$ - $\beta$-d-mannuronic acid (M) and $\alpha$-L-guluronic acid (G) residues, arranged in a block-wise fashion which has been widely used in CDR system ${ }^{[8]}$. It has gelation property in aqueous media due to the existence of divalent cations like $\mathrm{Ca}^{2+}$. SA can even be cross-linked covalently utilizing glutaraldehyde ${ }^{[9]}$. The crosslinking process of the polymer causes thickens and roughens of the polymer ${ }^{[10]}$. The development of IPNs of SA is fascinating because, by definition, IPNs contain two polymers which give a three-dimensional network, delivering free volume for simple encapsulation of medications.

The normal methods for synthesis of graft polysaccharides remains as conventional method (use of chemical-free radical initiator), high energy radiations method (gamma and X-ray), UV-radiation based method and Microwave based methods ${ }^{[11]}$, due to lack of availability of commercial method for the synthesis of the graft copolymers. In Conventional method, one initiator (e.g., ceric ammonium nitrate (CAN)) is vital for synthesis which generates free radical sites on the backbone polymer, where the monomer of graft will be added. It is not reasonable for commercial production scale synthesis. High energy radiation method is also not fit as high radiation, causing damage to the site of backbone of the polymer (radiolysis). In the UV-rays method, only surface grafting is done due to the low penetration of UV-rays. Consequently, microwave radiation rationally found to the best technique for uniting copolymer which creates free radical sites on the backbone polymer. It likewise guarantees high control and reproducibility of percentage grafting. Microwave radiation method is ordered into two sorts: Microwave initiated synthesis (MIS) and Microwave assisted synthesis (MAS) $)^{[12]}$. In MIS, the microwave itself creates free radical on the site of the polysaccharide backbone chain, from where the graft chains grow. In the other hand MAS, a chemical free radical initiator is used in microwave radiation to create free radical on the site of polysaccharide backbone chain, from where the graft chains grow ${ }^{[13]}$.

The objective of the present work revolves around synthesizing a modified tailored polymer by grafting PAM with GA, a natural polymer, as polyacrylamideg-Gum acacia (PAM-g-GA) by microwave-assisted synthesis, which is proposed to give a $\mathrm{pH}$-dependent activity to the grafted copolymer after alkaline hydrolysis and to develop IPN beads composed of the derivatized polymer with SA. Gabapentin (GBP), taken as a model drug, is a synthetic analogue of the GammaAmino Butyric Acid with anticonvulsant activity ${ }^{[14]}$. It decreases central nervous system disorganized electrical activity. It is used as adjunctive therapy in the management of epilepsy and for neuropathic pain syndromes ${ }^{[15]}$. This agent also exhibits analgesic properties. The reason for choosing gabapentin as a model drug to be encapsulated in $\mathrm{pH}$ responsive hydrogel beads lies in the consideration that gabapentin is absorbed primarily in the upper small intestine ( $\mathrm{pH}$ 6.6) via an active uptake transporter, which shows saturation dependent response at different clinically relevant doses ${ }^{[16]}$. The research work mainly focused on grafting PAM with GA as PAM-g-GA by microwaveassisted synthesis and to develop IPN hydrogel beads composed of the derivatized polymer with SA and to evaluate the beads for encapsulation of GBP and to study the release behavior of GBP from the IPN beads in different environment of GIT.

\section{MATERIALS AND METHODS}

GBP was purchased from TCI, Japan. PAM was purchased from Sigma- Aldrich, Bangalore. Ceric ammonium nitrate (CAN) was obtained from SRL Pvt. Ltd., Maharashtra. GA, SA, Calcium chloride $\left(\mathrm{CaCl}_{2}\right)$, Di-Sodium hydrogen Phosphate $\left(\mathrm{Na}_{2} \mathrm{HPO}_{4}\right)$, Potassium di-hydrogen phosphate $\left(\mathrm{KH}_{2} \mathrm{PO}_{4}\right)$, Sodium chloride $(\mathrm{NaCl})$, Potassium chloride $(\mathrm{KCl})$ and Hydrochloric acid $(\mathrm{HCl})$ were procured from SDFCL, Mumbai. Methanol was purchased from FCL, Ahmedabad. Distilled water was utilized all through the analysis. All the reagents were utilized minus any additional purging.

\section{Synthesis of tailored grafted copolymer:}

PAM-g-GA grafted copolymer was prepared by MAS method using CAN as an initiator followed by the method described earlier with small modification ${ }^{[17,18]}$. 
An amount equivalent to $4 \mathrm{~g}$ of PAM was dissolved in $60 \mathrm{ml}$ water by stirring for $30 \mathrm{~min} .1 \mathrm{~g}$ of GA was dissolved in $40 \mathrm{ml}$ distilled water and was added to the above solution. They were blended well and were moved to the response vessel ( $250 \mathrm{ml}$ borosil beaker) with the addition of the desired amount of CAN. It was transferred on the rotatable of a microwave oven and microwave irradiation at $800 \mathrm{~W}$ of power was carried out. Periodically, the irradiation was paused at the onset of boiling $\left(\sim 70^{\circ}\right)$ and was cooled in ice-cold water, to keep the formation of the competing homopolymer at the minimum. Schematic representation of the reaction process has been shown in fig. 1 .

Microwave irradiation and cooling process were continued until a gel-like mass was formed and was kept undisturbed for 60 min for complete grafting of the mixture. After that, the mixture was shifted into methanol and kept for $1 \mathrm{~d}$. Then, the precipitate grafting copolymer was filtered and dried as shown in fig. 2 . This was followed by alkaline hydrolysis to convert the amide group to free carboxylic group within the grafted copolymer.

\section{Preparation of blank IPN hydrogel beads:}

The polymeric solution of PAM-g-GA and SA at $(1: 1,1: 2$ and $2: 1$ ratio $\mathrm{w} / \mathrm{w})$ was prepared in distilled water with continuous stirring for $4 \mathrm{~h}$. This polymeric dispersion was stacked into $20 \mathrm{ml}$ hypodermic needle and gradually extruded into an aqueous solution of $\mathrm{CaCl}_{2}$ through a $\# 23$ needle while mixing. The acquired beads were separated from the $\mathrm{CaCl}_{2}$ solution, washed multiple times with $50 \mathrm{ml}$ distilled water, kept at room temperature for $24 \mathrm{~h}$ and afterward dried at $40^{\circ}$ for $12 \mathrm{~h}^{[19]}$.
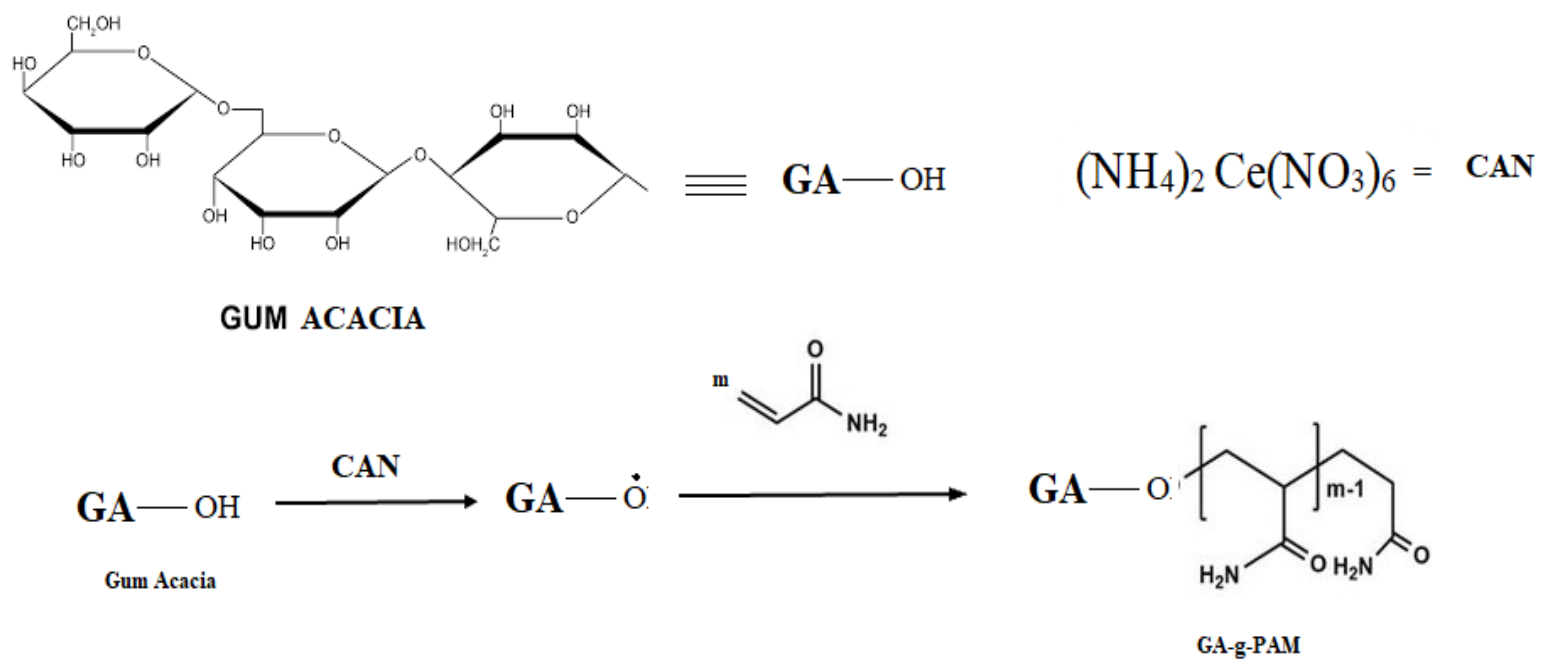

Fig. 1: Schematic pathway of Synthesis of tailored grafted co-polymer

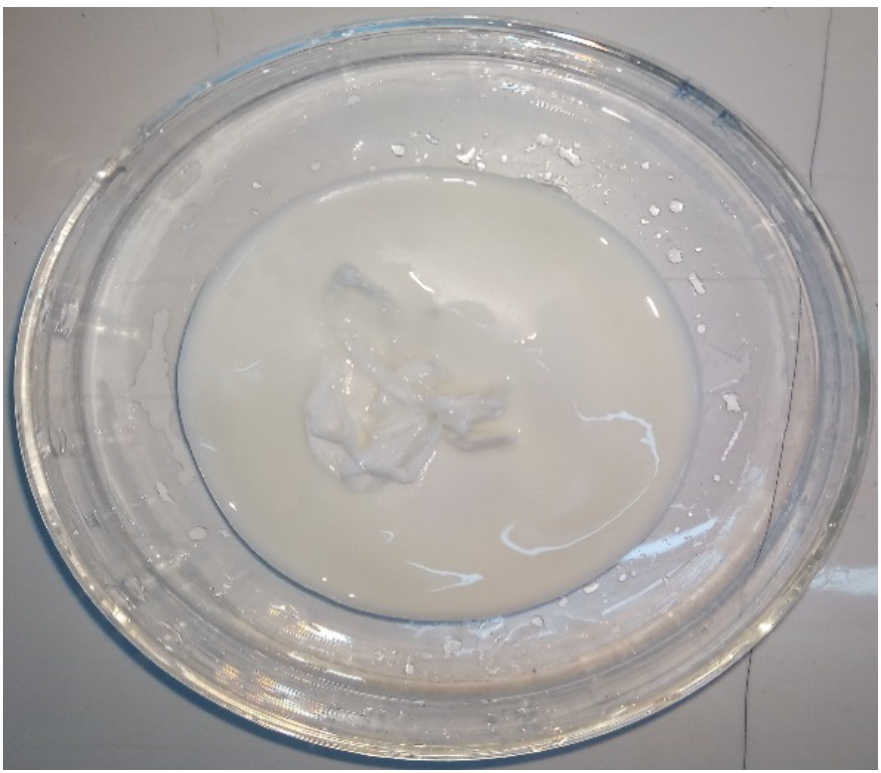

Fig. 2: Microwave Irradiation Synthesis of GA-g-PAM copolymer 
Preparation of drug loaded IPN hydrogel beads:

The polymeric solution of PAM-g-GA and SA at (1:1, $1: 2$ and $2: 1$ ratio $\mathrm{w} / \mathrm{w}$ ) was prepared in distilled water with continuous stirring for $4 \mathrm{~h}$. An exactly weighed quantity of GBP was added into the polymeric solution and mixed properly on a magnetic stirrer for $30 \mathrm{~min}$. This polymeric dispersion was loaded into $20 \mathrm{ml}$ hypodermic syringe and slowly extruded into an aqueous solution of $\mathrm{CaCl}_{2}$ through a $\# 23$ needle while stirring. The obtained microbeads were removed from the $\mathrm{CaCl}_{2}$ solution; washed two times with $50 \mathrm{ml}$ distilled water, kept at room temperature for $24 \mathrm{~h}$ and then dried at $40^{\circ}$ for $12 \mathrm{~h}$ as shown in fig. 3. Table 1 contains the various compositions of IPN microbeads ${ }^{[20]}$.

\section{CHARACTERIZATION}

\section{Fourier-transform infrared (FTIR) analysis:}

The FTIR spectra of GA, PAM-g-GA, PAM, blank IPN beads and drug-loaded IPN beads were recorded using ATR- FTIR spectrophotometer (Agilent Technology, CDR-630). They were scanned in the range of $500 \mathrm{~cm}^{-1}$ and $4000 \mathrm{~cm}^{-1}$ at a resolution of $12 \mathrm{~cm}$ at $25^{\circ[21]}$.

\section{Scanning electron microscope (SEM) Analysis:}

The surface morphology and shape of the blank IPN beads and drug-loaded IPN beads were evaluated by the method described Chirila et al. ${ }^{[22]}$. Before analysis, the samples were mounted onto stubs using doublesided dried carbon tape and vacuum coated with goldpalladium film (thickness $2 \mathrm{~nm}$ ) using sputter coater which makes them electrically conductive.
X-Ray Diffraction (XRD) analysis:

The XRD of PAM-g-GA, GBP, blank IPN beads and drug-loaded IPN beads were studied by using diffractometric system (Rigaku, Japan, SmartLab 9kW) at $1.5 \mathrm{~mA}$ and $30 \mathrm{KV}$ over the range $2 \theta=5^{\circ}$ to $80^{\circ}$ at a rate of $2 \theta=5^{\circ} / \mathrm{min}^{[23]}$.

\section{Differential scanning calorimetry (DSC) analysis:}

PAM-g-GA, PAM, GBP, blank IPN beads and drugloaded IPN beads were assessed by DSC analyzer (Perkin Elmer- USA, JADE DSC). Aliquot of $10 \mathrm{mg}$ of dried samples were taken in an alumina crucible and heated from $10-400^{\circ} \mathrm{C}$, at a heating rate $10^{\circ} \mathrm{C} / \mathrm{min}$ with a continuous flow of nitrogen ${ }^{[24]}$.

\section{Percentage of drug entrapment efficiency (DEE):}

$50 \mathrm{mg}$ accurately weighed IPN beads were immersed in $50 \mathrm{ml}$ buffer solution of $\mathrm{pH} 1.2, \mathrm{pH} 6.8$ and $\mathrm{pH} 7.4$ separately for $24 \mathrm{~h}$. Then, the samples were shaken for $5 \mathrm{~min}$ and filtered. The filtered solution was analyzed by using UV Spectrophotometer (Shimadzu, 1800) and calculated the percentage $(\%)$ of $\mathrm{DEE}^{[25]}$.

\section{Loose surface crystal study (LSC):}

The LSC characteristic of IPN beads was determined by the method described Chakraborty et al. ${ }^{[26]} .50 \mathrm{mg}$ of beads were suspended in $50 \mathrm{ml}$ of buffer solution of $\mathrm{pH} 1.2, \mathrm{pH} 6.8$ and $\mathrm{pH} 7.4$ separately and were shaken for $15 \mathrm{~min}$ in a mechanical shaker. The amount of drug separated from the surface was analyzed by UV-Spectrophotometer. Percentage of drug present
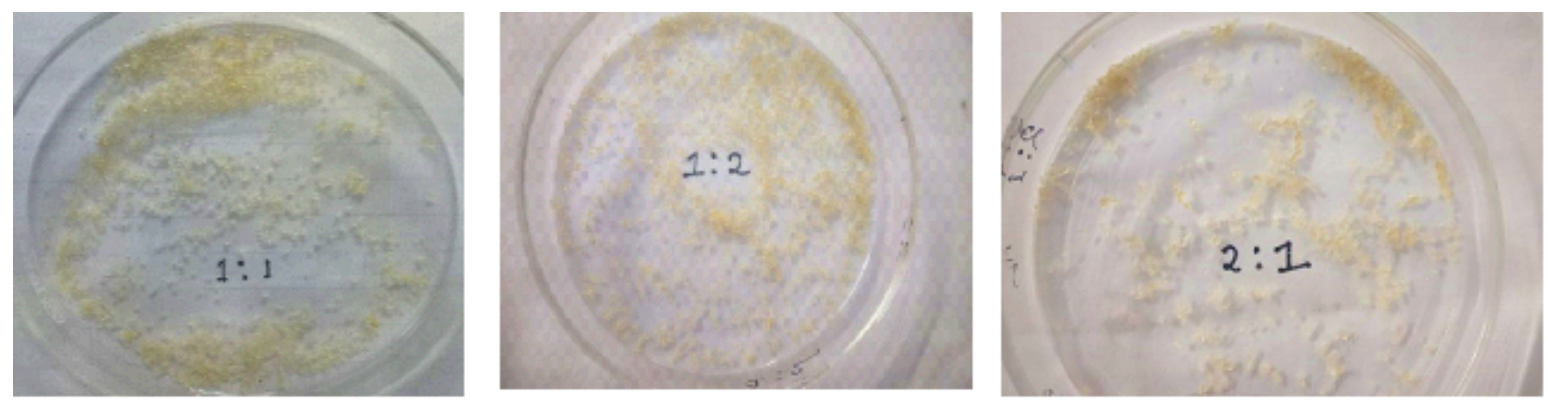

Fig. 3: Preparation of GBP loaded IPN Hydrogel beads of GA-g-PAM and SA at (1:1, 1:2 and 2:1) ratio w/w

TABLE 1: COMPOSITION OF IPN MICROBEADS

\begin{tabular}{lcccc}
\hline Codes & GA-g-PAM $(\% \mathrm{w} / \mathrm{v})$ & $\mathrm{SA}(\% \mathrm{w} / \mathrm{v})$ & Gabapentin $(\% \mathrm{w} / \mathrm{v})$ & $\mathrm{CaCl}_{2}(\% \mathrm{w} / \mathrm{v})$ \\
\hline F1 & 1 & 1 & - & 8 \\
F2 & 1 & 2 & - & 8 \\
F3 & 2 & 1 & - & 8 \\
F4 & 1 & 1 & 50 & 8 \\
F5 & 1 & 2 & 50 & 8 \\
F6 & 2 & 1 & 50 & 8 \\
\hline
\end{tabular}


loosely on the surface of the beads was calculated by the following equation.

$\%$ LSC with respect to weight of microbeads=amount of drug $(\mathrm{mg})$ released after $15 \mathrm{~min} /$ total weight of microbeads used in the experiment $\times 100 \%$ LSC with respect to entrapped drug=amount of drug (mg) released after $15 \mathrm{~min} /$ drug content of microbeads used in the experiment $\times 100$.

\section{pH dependent swelling behavior of IPN beads:}

The swelling index of IPN beads at different $\mathrm{pH}$ was characterized by the method described by Rao et al. ${ }^{[27]}$. Required amount dried beads were transferred in 20 $\mathrm{ml}$ buffer solution of $\mathrm{pH} 1.2, \mathrm{pH} 6.8$ and $\mathrm{pH} 7.4$ at room temperature. In the specific time, the beads were withdrawn from the solution and excess solution on the surface of beads was removed by the gentle touch of tissue paper. Then weight measurement the swollen sample were done and calculated the swelling index (SI) using the formula, SI=Weight of swollen sample $\left(\mathrm{W}_{\mathrm{s}}\right)$-Initial weight of the dried sample $\left(\mathrm{W}_{\mathrm{d}}\right) /$ weight of the dried sample $\times 100$

\section{In vitro drug release:}

The drug release study was performed by USP type I dissolution apparatus (Electrolab, TDL-08L). The dissolution medium was $\mathrm{pH} 1.2, \mathrm{pH} 6.8$ or $\mathrm{pH} 7.4$ phosphate buffer. Exactly, a $100 \mathrm{mg}$ of drug-loaded beads are transferred into the basket, the temperature of dissolution medium was kept at $37 \pm 0.5^{\circ}$ and a stirring rate of $100 \mathrm{rpm}$ was maintained. The samples were withdrawn at predetermined time intervals and immediately the same volume of fresh buffer was replaced. After suitable dilution, the sample was assayed spectrophotometrically at $266 \mathrm{~nm}^{[28]}$.

\section{RESULTS AND DISCUSSION}

We have a PAM-g-GA copolymer synthesized by a microwave-assisted method. Microwave irradiation is used in conjunction with CAN to produce freeradical sites on the GA structure. CAN has an electron deficiency that can capture electrons from the GA's hydroxyl group to form a new $\mathrm{Ce}-\mathrm{O}$ bond. As a result, the Ce-O bond formed is polar than the $\mathrm{O}-\mathrm{H}$ bond and can break by coverage to microwave irradiation subsequently forming free radical sites on the GA backbone which acts as a point of growth for grafting chains. Alkaline hydrolysis of the synthesized grafting co-polymer was performed using standard sodium hydroxide for transfer it to a $\mathrm{pH}$-sensitive carrier. $\mathrm{By}$ means of alkaline hydrolysis, $-\mathrm{CONH}_{2}$ groups of PAM transform into - COONa which converts it into a $\mathrm{pH}$ sensitive copolymer.

During the preparation of beads, as soon as the solution (PAM-g-GA)-Na Alginate comes into contact with $\mathrm{Ca}^{2+}$ cations $\left(\mathrm{CaCl}_{2}\right.$ solution), the ionic cross-linking between two polymer chains will be formed. The exchange of $\mathrm{Na}^{+}$with the polymer occurs with $\mathrm{Ca}^{+2}$ ions. These $\mathrm{Ca}^{+2}$ are ionically replaced at the carboxylate site and the second strand of Na Alginate or PAM-g-GA can also be connected to $\mathrm{Ca}^{2+}$ forming a link in which cations are attached to two or three Na Alginate or PAM-g-GA strands together to form spherical IPNs.

FTIR Spectra of GBP, GA, PAM, PAM-g-GA, blank IPN beads and drug-loaded IPN beads are illustrated in fig. 4. In GA $2919 \mathrm{~cm}^{-1}$ and broad peaks at $3420 \mathrm{~cm}^{-1}$ are attributed to stretching vibration of $\mathrm{C}-\mathrm{H}$ and $\mathrm{O}-\mathrm{H}$, respectively. In PAM, peaks of amide-I (1619 cm-1), $\mathrm{CH}_{2}$ scissoring $\left(1457 \mathrm{~cm}^{-1}\right)$ and $\mathrm{C}-\mathrm{N}\left(1457 \mathrm{~cm}^{-1}\right)$ stretching vibrations are observed with several other identical peaks. In PAM-g-GA, the small peak at

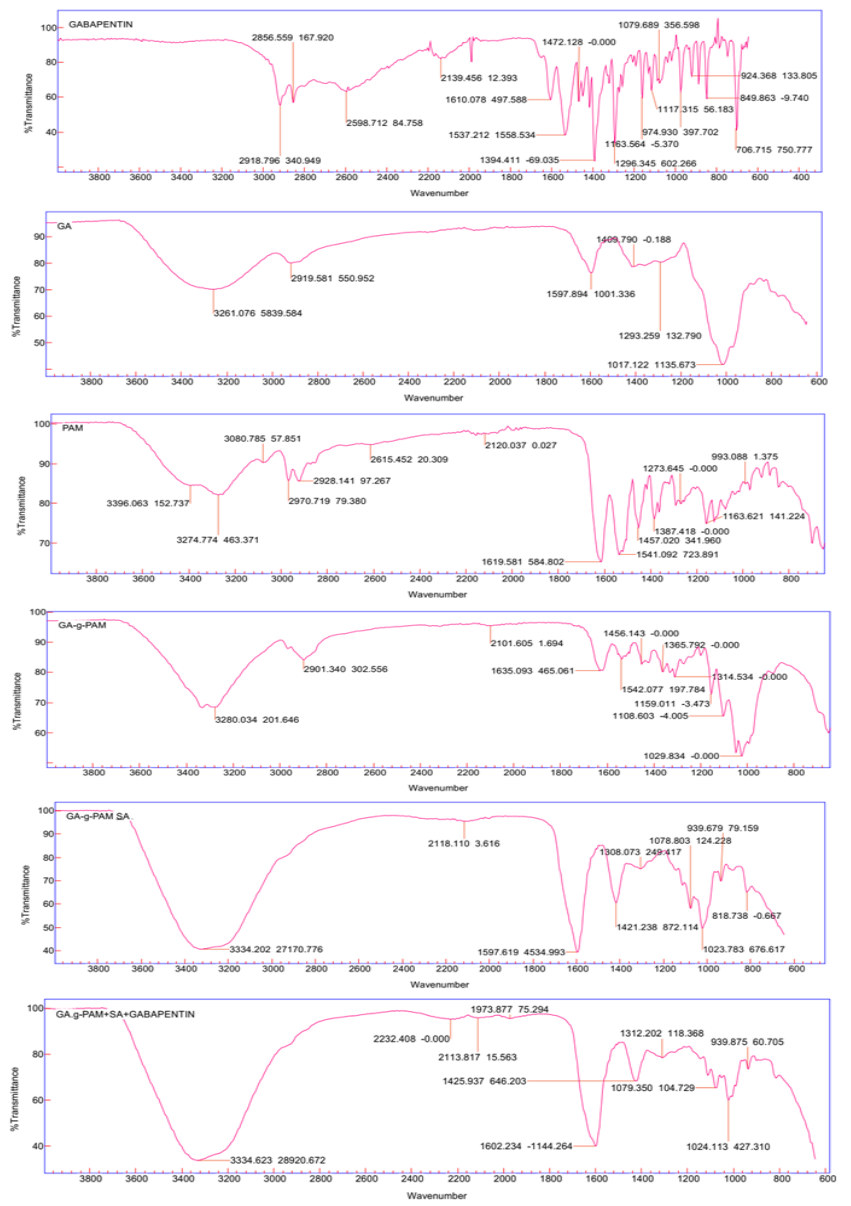

Fig. 4: FTIR Spectra of pure drug GBP, GA, PAM, GA-g-PAM, blank IPN beads and drug-loaded beads 
$2901 \mathrm{~cm}^{-1}$ and broad peaks near at $3410 \mathrm{~cm}^{-1}$ are attributed to stretching vibration of $\mathrm{C}-\mathrm{H}$ and $\mathrm{O}-\mathrm{H}$, respectively. The peaks at $1108 \mathrm{~cm}^{-1}$ and $1029 \mathrm{~cm}^{-1}$ are due to the ether linkage formed by the reaction between -OH group of GA and PAM. Some more peaks of amide-I $\left(1635 \mathrm{~cm}^{-1}\right)$, amide II $\left(1610 \mathrm{~cm}^{-1}\right)$, $\mathrm{CH}_{2}$ scissoring $\left(1456 \mathrm{~cm}^{-1}\right), \mathrm{C}-\mathrm{N}\left(1414 \mathrm{~cm}^{-1}\right)$ stretching vibrations confirm the grafting of PAM chains onto GA backbone. In GBP, a combination of bending vibration of $\mathrm{C}-\mathrm{O}, \mathrm{C}-\mathrm{N}$ and $\mathrm{C}-\mathrm{C}$ from $1350 \mathrm{~cm}^{-1}$ to $900 \mathrm{~cm}^{-1}$. Stretching vibration of a carboxylic group from $1320 \mathrm{~cm}^{-1}$ to $1210 \mathrm{~cm}^{-1}$. Stretching vibration of $\mathrm{C}=\mathrm{C}$ and bending vibration of $\mathrm{O}-\mathrm{H}$ from $1650 \mathrm{~cm}^{-1}$ to $1450 \mathrm{~cm}^{-1}$. Bending vibration of $\mathrm{N}-\mathrm{H}$ from 1640 $\mathrm{cm}^{-1}$ to $1550 \mathrm{~cm}^{-1}$. Stretching vibration of ammonium near $2100 \mathrm{~cm}^{-1}$. Stretching vibration of C-H aliphatic from $3100 \mathrm{~cm}^{-1}$ to $2600 \mathrm{~cm}^{-1}$. In blank IPN beads, stretching vibration of $\mathrm{CH}_{2}-\mathrm{O}-\mathrm{CH}_{2}$ from $1023 \mathrm{~cm}^{-1}$ and $1079 \mathrm{~cm}^{-1} .1420 \mathrm{~cm}^{-1}$ is attributed to the stretching vibration of C-N. The broad peaks near at $3322 \mathrm{~cm}^{-1}$ are due to stretching vibration of $\mathrm{O}-\mathrm{H}$. All the principal peak of GBP were present in drug-loaded IPN beads with minor differences in frequency which indicates there is no interaction between polymer and drug. The shift of bands in beads observed, indicated the IPN structures of is formed between two polymers ${ }^{[29]}$.

SEM micrograph of blank IPN beads and drug-loaded IPN beads were taken at different magnification as shown in fig. 5. The surface morphology of blank beads was smooth surface and less spherical shape. While the drug loaded IPN beads were slightly rough surface and predominantly spherical in shape due to shrinkage of the beads during the drying process ${ }^{[30]}$.

XRD analysis gives a hint about the crystallinity of drug in the IPN beads. Fig. 6 shows the XRD patterns of GBP, PAM-g-GA, blank IPN beads and drug-loaded IPN beads. Here, in the XRD the GBP, sharp peaks were noticed due to the crystalline nature of GBP. These peaks are not observed in GBP loaded beads and blank beads. This indicates that GBP particles are distributed uniformly inside the polymer matrix during drug loading in drug loaded beads. So that, any crystalline nature of drug was not observed in drug loaded IPN beads. The sharp peaks at $2 \theta$ of 35 and $45^{\circ}$ indicated a little amount of drug present in the surface of the beads which may relate with the amount of $\mathrm{LSC}^{[31]}$.

Fig. 7, showing the DSC thermograms of PAM, PAMg-GA, GBP, blank IPN beads and drug-loaded IPN beads. PAM exhibits a peak at $228^{\circ}$. The endothermic peaks of PAM-g-GA are shifted from $228^{\circ}$ to $249^{\circ}$ which indicate PAM and GA mixed uniformly. The sharp endothermic peaks of GBP were observed at $178^{\circ}$ which indicates the melting point of GBP. The blank IPN beads have shown two endothermic peaks at $108^{\circ}$ and $198^{\circ}$. There are two noticeable endothermic peaks of drug loaded IPN beads which is shift from $108^{\circ}$ and $198^{\circ}$ to $121^{\circ}$ and $205^{\circ}$ respectively. Changes in the endothermic peaks in beads may be due to formation of IPN structure including sodium alginate doe to chain entanglements. The characteristic peak of the drug was
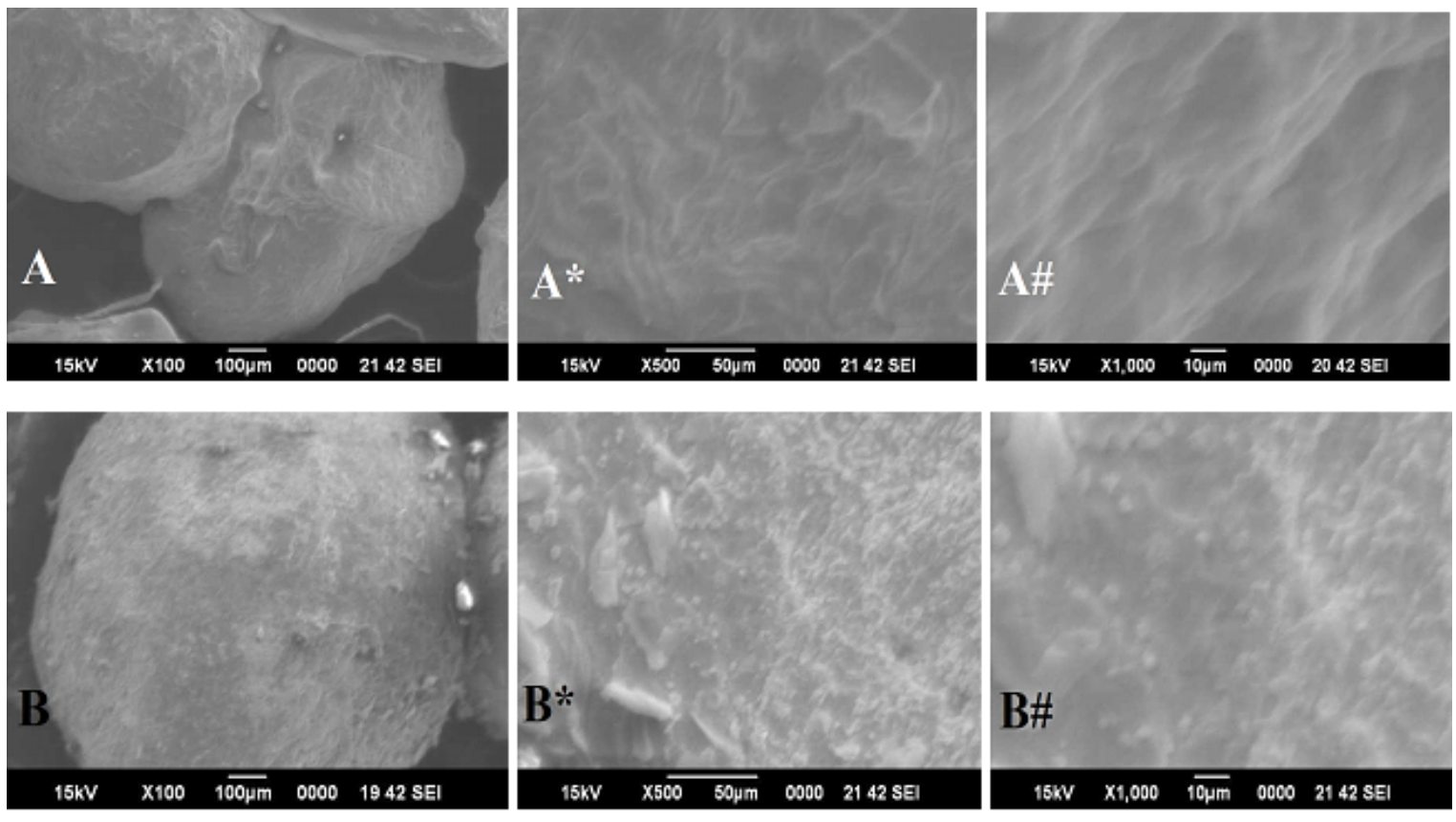

Fig. 5: SEM Images of F2 (1:2) IPN beads (A) and F5 (1:2) IPN beads (B). A*; A\# and B*; B\# corresponds to their surface in different magnification 


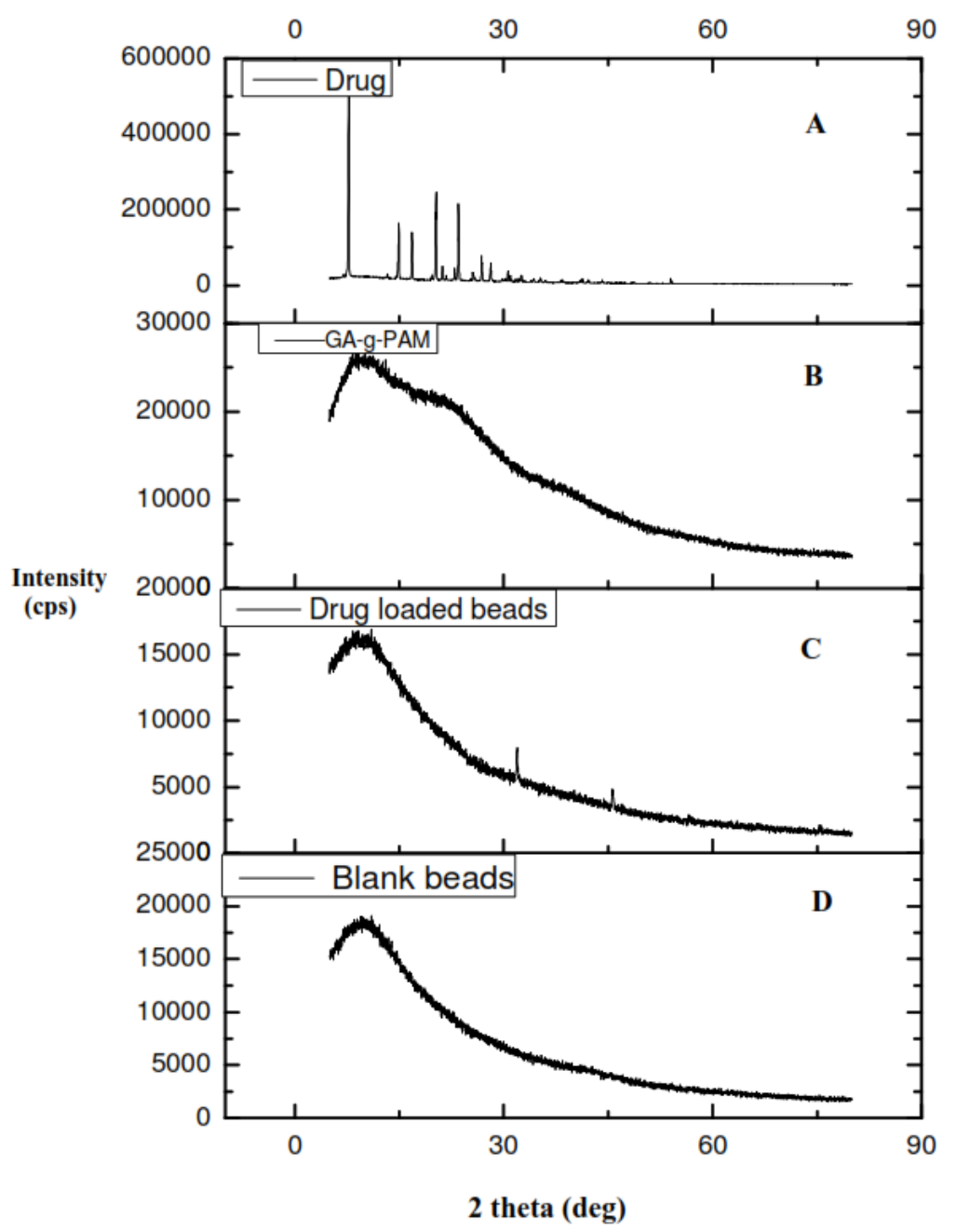

Fig. 6: XRD spectra of (A) pure drug GBP, (B) GA-g-PAM, (C) drug-loaded beads and (D) blank IPN beads

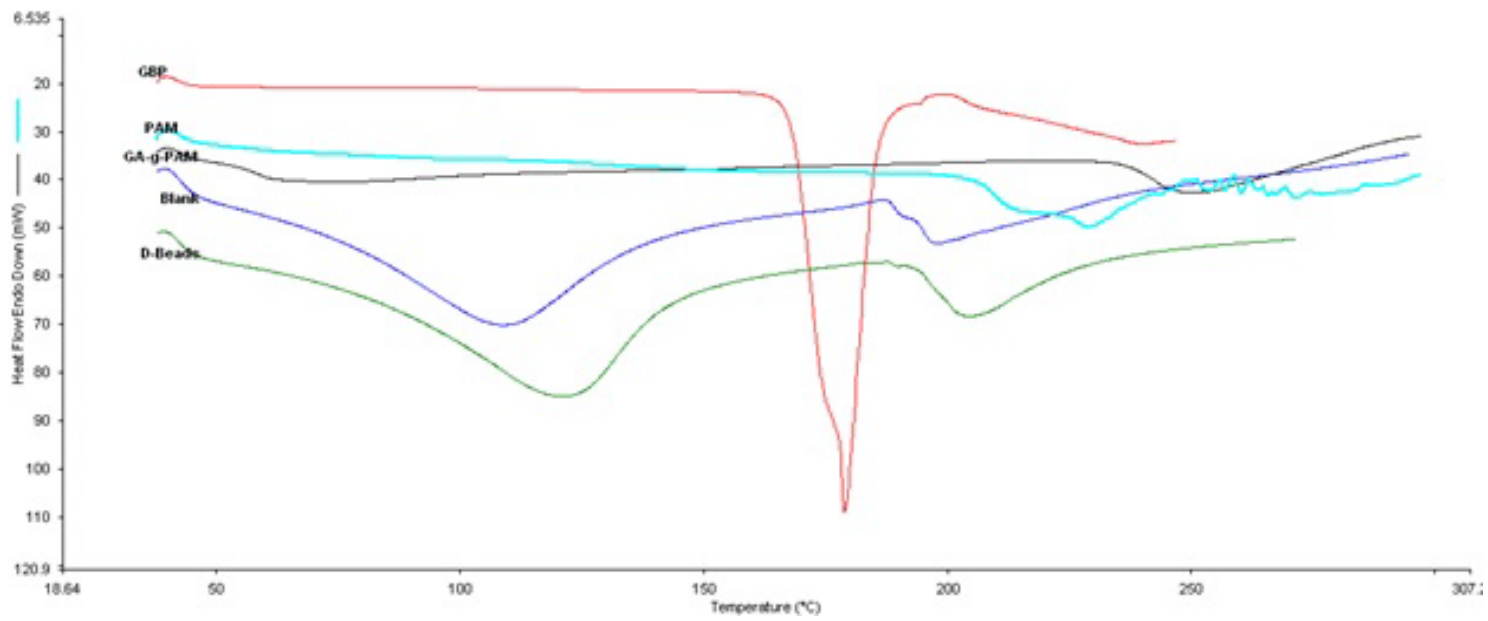

Fig. 7: DSC spectra of pure drug GBP, PAM, GA-g-PAM, blank IPN beads and drug-loaded beads

not observed in DSC thermograms of the drug-loaded IPN beads, confirming the dispersion of drug in the beads at the molecular level ${ }^{[32]}$.

The DEE of drug-loaded IPN beads were found to be in the range of $36.85 \%$ to $40.35 \%$ (Table 2 ). IPN beads with 1:2 ratio of PAM-g-GA: SA has shown higher DEE than the 2:1 ratio of PAM-g-GA: SA. In case of 2:1 ratio, the polymer matrix might be loosely network and have large orifice which results in leakage of drugs into the gelation medium due to the amount of grafting 
polymer higher than the SA, which lower the $\mathrm{DEE}^{[33]}$.

LSC is an important parameter which indicates the drug amount present on the surface of the IPN beads for rapid absorption and onset of action. A very little amount of drug varies from $0.928 \%$ to $6.383 \%$ are available on the surface of beads, shown in Table $2^{[34]}$.

The outcome of the swelling study is summarized in fig. 8. These studies confirmed that the swelling behavior of the sample was changed when the $\mathrm{pH}$ media different. The percentage of swelling in $\mathrm{pH} 1.2, \mathrm{pH} 6.8$ and $\mathrm{pH}$ 7.4 are shown in Table 3. The beads swelled in $\mathrm{pH} 6.8$ more than in $\mathrm{pH}$ 7.4. But in $\mathrm{pH} 1.2$ the beads were not swollen. The surface of beads in $\mathrm{pH} 1.2$ was plain, but the surface of beads in $\mathrm{pH} 6.8$ solution was spongy than the surface of beads in $\mathrm{pH}$ 7.4. It is significant that at $\mathrm{pH}$ 6.8 the cross-linked grafted polymer is ionized which allow water to enter into beads.

The in vitro dissolution study is shown in fig. 9. The formulation coded as F4 (1:2), F5 (1:2) and F6 (1:2) shown a drug release of $54 \%, 91 \%$ and $69 \%$ consecutively, after $7 \mathrm{~h}$. The result of the kinetic data was obtained by fitting the value into several mathematical models. The regression coefficient $\left(\mathrm{R}^{2}\right)$ value obtained of the optimized formulation (F5) for the zero-order,

TABLE 2: DRUG ENTRAPMENT EFFICIENCY AND LOOSE SURFACE CRYSTAL STUDY OF IPN MICROBEADS

\begin{tabular}{lcccc}
\hline pH & Formulation & \% DEE & LSC (\%) to gross wt. of the microbeads & LSC (\%) with respect to entrapped drug \\
\hline $\mathrm{pH} 1.2$ & F4 (1:1) & 38.85 & 0.9285 & 4.8162 \\
& F4 (1:2) & 40.14 & 1.2142 & 6.1513 \\
& F4 (2:1) & 37.78 & 0.9285 & 4.6615 \\
$\mathrm{pH} 6.8$ & F5 (1:1) & 39.21 & 1 & 5.102 \\
& F5 (1:2) & 40.35 & 1.1428 & 5.6858 \\
& F5 (2:1) & 38.57 & 1.0714 & 5.3976 \\
$\mathrm{pH} 7.4$ & F6 (1:1) & 38.14 & 1.1428 & 5.7778 \\
& F6 (1:2) & 39.57 & 1.4285 & 7.1428 \\
& F6 (2:1) & 36.85 & 1.2857 & 6.3838 \\
\hline
\end{tabular}
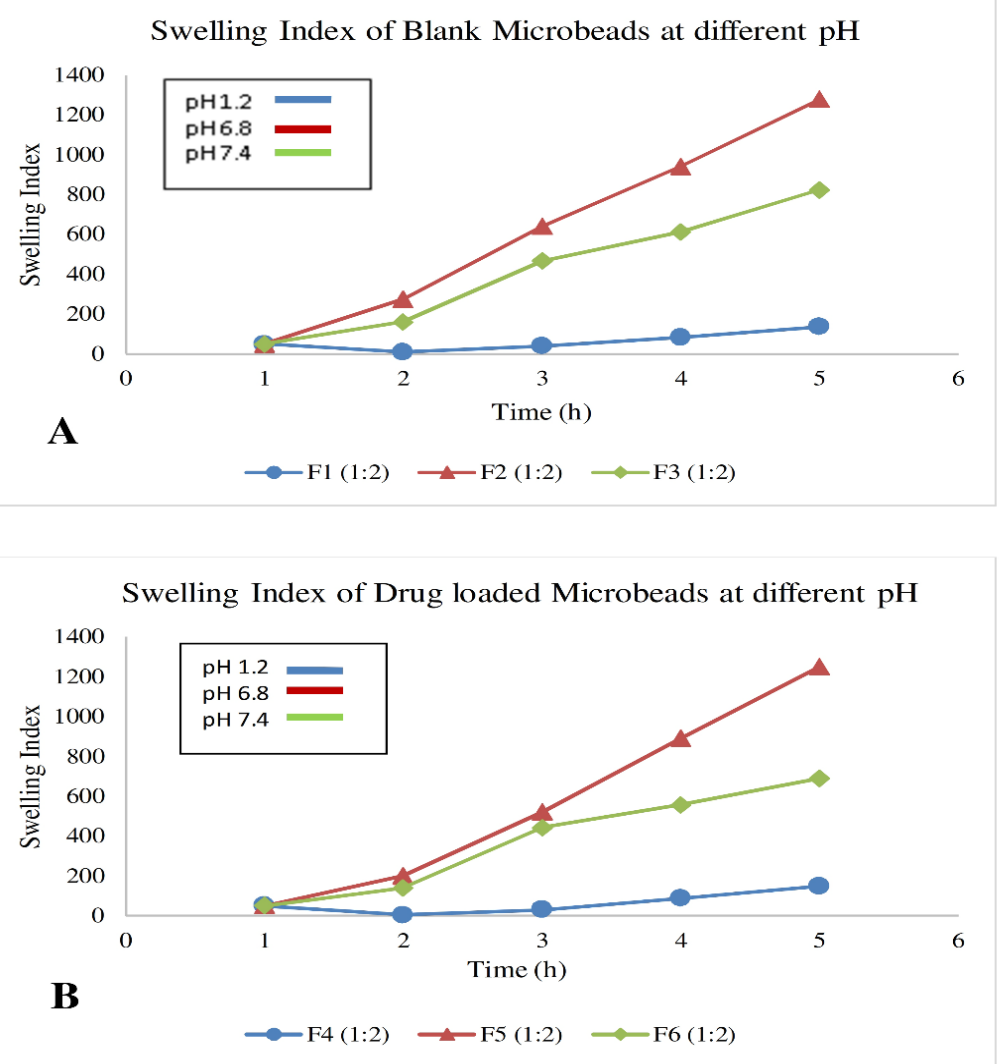

Fig. 8: Swelling Index of (A) blank IPN microbeads and (B) drug-loaded IPN microbeads at different pH with the different time interval 
TABLE 3: SWELLING INDEX AT DIFFERENT PH WITH DIFFERENT TIME INTERVAL

\begin{tabular}{lcccccc}
\hline pH & Formulation & Initial weight $(\mathbf{m g})$ & SI (1h) & SI (2h) & SI (3h) & SI (overnight) \\
\hline \multirow{2}{*}{ pH 1.2 } & F1 (1:2) & 50 & 10 & 40 & 82 & 136 \\
& F4 (1:2) & 50 & 4 & 30 & 88 & 148 \\
pH 6.8 & F2 (1:2) & 50 & 274 & 640 & 940 & 1278 \\
& F5 (1:2) & 50 & 200 & 520 & 890 & 1250 \\
pH 7.4 & F3 (1:2) & 50 & 160 & 466 & 612 & 824 \\
& F6 (1:2) & 50 & 140 & 442 & 556 & 690 \\
\hline
\end{tabular}

Dissolution profile of all Formulation

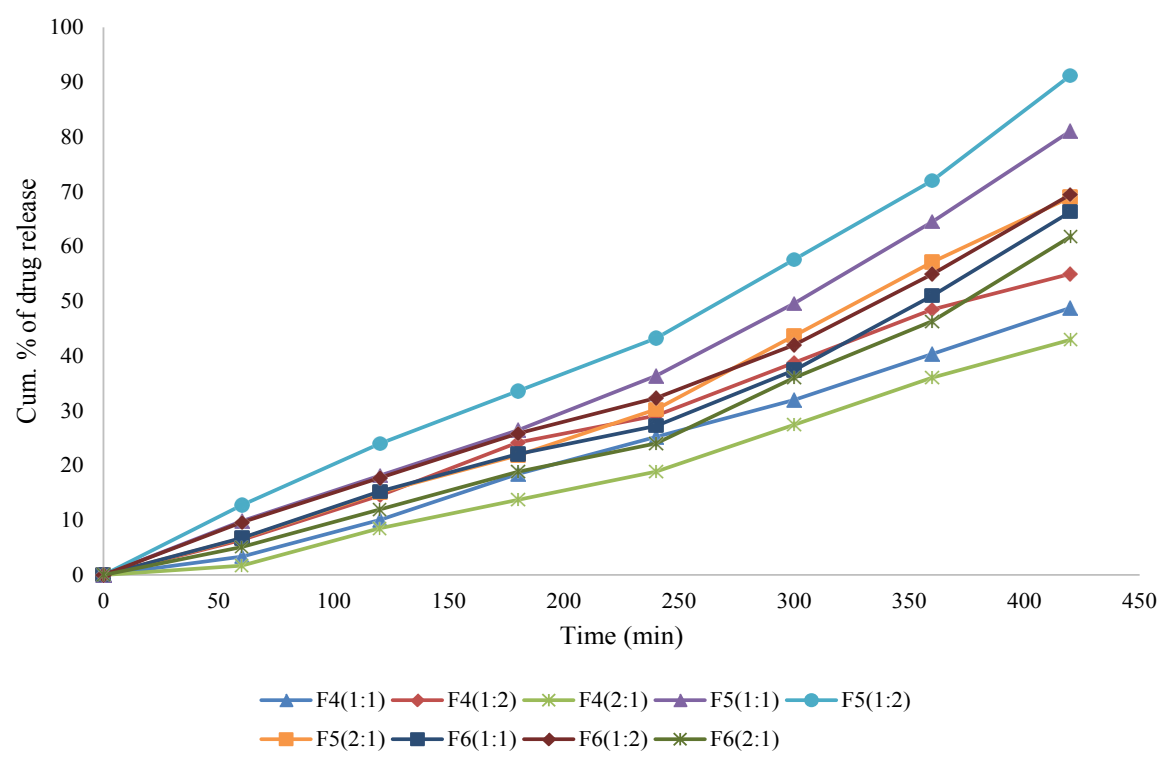

Fig 9: In vitro drug release plot

first-order, Higuchi and Hixson-Crowell were 0.98, $0.96,0.94$ and 0.99 , respectively.

Among all the release models, Korsmeyer-Peppas model was found best fit $\left(\mathrm{R}^{2}=0.99\right)$ with an exponent $(\mathrm{n}=0.9)$ indicating Non-Fickian release kinetic (anomalous diffusion) of GBP from IPN microbeads.

This anomalous diffusion of the drug release demonstrated a mutual effect of swelling and diffusioncontrolled drug release mechanism.

The $\mathrm{pH}$-responsive IPN beads of PAM-g-GA and SA were developed by crosslinking method for gastro-protective controlled delivery of GBP. The $\mathrm{pH}$-responsive PAM-g-GA was synthesized by the microwave-assisted method and characterized by FTIR spectra, DSC analysis and XRD analysis. Three different formulations were prepared by different ratio of PAMg-GA and SA (1:1, 1:2 and 2:1). The $\mathrm{pH}$-responsive activity of the IPN microbead in $\mathrm{pH} 6.8$ is more than in $\mathrm{pH}$ 7.4. But in $\mathrm{pH} 1.2$ the beads were not swollen. The release of GBP from IPN beads was slow in $\mathrm{pH}$ 1.2. In $\mathrm{pH} 6.8$, the release of the drug was faster than in $\mathrm{pH}$ 7.4. It was concluded that F5 (1:2) formulation shown satisfactory result having cumulative percentage drug release was $91 \%$ in phosphate buffer $\mathrm{pH}$ 6.8. The mechanism of GBP release from the IPN was assumed to be governed by the mutual effect of swelling and diffusion due to its $\mathrm{pH}$-responsive swelling property.

\section{Acknowledgements:}

The authors offer there sincere thanks to the Management, Himalayan Pharmacy Institute for providing the research facility to carry out the work. The authors are thankful to the Department of Pharmaceutical Sciences; Dibrugarh University, Assam, India, for providing DSC facility. The authors are thankful to Central Instrumentation Facility, Birla Institute of Technology, Mesra, Ranchi, India for providing XRD and SEM facility.

\section{Conflict of interests:}

The authors declared no conflicts of interest.

\section{REFERENCES}

1. Matricardi P, Di Meo C, Coviello T, Hennink WE, Alhaique F. Interpenetrating polymer networks polysaccharide hydrogels for drug delivery and tissue engineering. Adv Drug Deliv Rev 
2013;65(9):1172-87.

2. Kulkarni RV, Sreedhar V, Mutalik S, Setty CM, Sa B. Interpenetrating network hydrogel membranes of sodium alginate and poly (vinyl alcohol) for controlled release of prazosin hydrochloride through skin. Int J Biol Macromol 2010;47(4):520-7.

3. Kulkarni RV, Mutalik S, Mangond BS, Nayak UY. Novel interpenetrated polymer network microbeads of natural polysaccharides for modified release of water soluble drug: in vitro and in vivo evaluation. J Pharm Pharmacol 2012;64(4):530-40.

4. Renard D, Lavenant-Gourgeon L, Ralet MC, Sanchez C. Acacia s enegal gum: continuum of molecular species differing by their protein to sugar ratio, molecular weight and charges. Biomacromolecules 2006;7(9):2637-49.

5. Cesàro A, Gamini A, Navarini L. Supramolecular structure of microbial polysaccharides in solution: from chain conformation to rheological properties. Polymer 1992;33(19):4001-8.

6. Hadjesfandiari N, Parambath A. Stealth coatings for nanoparticles: Polyethylene glycol alternatives. InEngineering of Biomaterials for Drug Delivery Systems 2018:345-61.

7. Polyacrylamide, chemical compound. Encyclopedia Britannica 2019.

8. Işiklan N. Controlled release of insecticide carbaryl from sodium alginate, sodium alginate/gelatin and sodium alginate/ sodium carboxymethyl cellulose blend beads crosslinked with glutaraldehyde. J Appl Polym Sci 2006;99(4):1310-9.

9. $\mathrm{Su} X, \mathrm{Chen} \mathrm{B.} \mathrm{Tough,} \mathrm{resilient} \mathrm{and} \mathrm{pH}$-sensitive interpenetrating polyacrylamide/alginate/montmorillonite nanocomposite hydrogels. Carbohydr Polym 2018;197:497-507.

10. Bregni C, Degrassi J, Garcia R, Lamas MC, Firenstein RDM. Alginate microspheres of Bacillus subtilis. Ars Pharmaceutica 2000;41:245-8.

11. McLean MJ. Gabapentin. Epilepsia 1995;36:73-86.

12. James o, McNamara. Drug effective in the therapy of the epilepsies, Goodman \& Gilman's: The Pharmacological basis of therapeutics, Chapter 21, Tenth Edition 2001:538-539.

13. Sen G, Pal S. Microwave initiated synthesis of polyacrylamide grafted carboxymethylstarch (CMS-g-PAM): application as a novel matrix for sustained drug release. Int J Biol Macromol 2009;45(1):48-55.

14. Rani P, Sen G, Mishra S, Jha U. Microwave assisted synthesis of polyacrylamide grafted gum ghatti and its application as flocculant. Carbohydr Polym 2012;89(1):275-81.

15. Mishra S, Mukul A, Sen G, Jha U. Microwave assisted synthesis of polyacrylamide grafted starch (St-g-PAM) and its applicability as flocculant for water treatment. Int J Biol Macromol 2011;48(1):106-11.

16. Mishra S, Sen G. Microwave initiated synthesis of polymethylmethacrylate grafted guar (GG-g-PMMA), characterizations and applications. Int $\mathrm{J}$ Biol Macromol 2011;48(4):688-94.

17. Mishra S, Rani GU, Sen G. Microwave initiated synthesis and application of polyacrylic acid grafted carboxymethyl cellulose. Carbohydr Polym 2012;87(3):2255-62.

18. Halder A, Maiti S, Sa B. Entrapment efficiency and release characteristics of polyethyleneimine-treated or-untreated calcium alginate beads loaded with propranolol-resin complex. Int J Pharm 2005;302(1-2):84-94.

19. Boppana R, Mohan GK, Nayak U, Mutalik S, Sa B, Kulkarni RV. Novel pH-sensitive IPNs of polyacrylamide-g-gum ghatti and sodium alginate for gastro-protective drug delivery. Int $\mathbf{J}$
Biol Macromol 2015;75:133-43.

20. Bashir S, Teo YY, Naeem S, Ramesh S, Ramesh K. pH responsive N-succinyl chitosan/Poly (acrylamide-co-acrylic acid) hydrogels and in vitro release of 5-fluorouracil. PloS one 2017;12:1-24.

21. Chirila TV, Vijayasekaran S, Horne R, Chen YC, Dalton PD, Constable IJ, et al. Interpenetrating polymer network (IPN) as a permanent joint between the elements of a new type of artificial cornea. J Biomed Mater Res 1994;28(6):745-53.

22. Rani M, Agarwal A, Negi YS. Characterization and biodegradation studies for interpenetrating polymeric network (IPN) of chitosan-amino acid beads. J Biomater Nanobiotechnol 2011;2(01):71.

23. Kajjari PB, Manjeshwar LS, Aminabhavi TM. Novel interpenetrating polymer network hydrogel microspheres of chitosan and poly (acrylamide)-grafted-guar gum for controlled release of ciprofloxacin. Ind Eng Chem Res 2011;50:13280-7.

24. Bhattacharya SS, Ghosh AK, Banerjee S, Chattopadhyay P, Ghosh A. $\mathrm{Al}^{3+}$ ion cross-linked interpenetrating polymeric network microbeads from tailored natural polysaccharides. Int J Biol Macromol 2012;51(5):1173-84.

25. Ghosh A, Chakraborty P. Formulation and mathematical optimization of controlled release calcium alginate micro pellets of frusemide. Biomed Res Int 2013;1-14.

26. Rao KK, Naidu BV, Subha MC, Sairam M, Aminabhavi TM. Novel chitosan-based $\mathrm{pH}$-sensitive interpenetrating network microgels for the controlled release of cefadroxil. Carbohydr Polym 2006;66(3):333-44.

27. Fernandez-Hervas MJ, Holgado MA, Fini A, Fell JT. In vitro evaluation of alginate beads of a diclofenac salt. Int J Pharm 1998;163(1-2):23-34.

28. Suo H, Zhang D, Yin J, Qian J, Wu ZL, Fu J. Interpenetrating polymer network hydrogels composed of chitosan and photocrosslinkable gelatin with enhanced mechanical properties for tissue engineering. Mater Sci Eng C 2018;92:612-20.

29. Kaity S, Ghosh A. Facile preparation of acrylamide grafted locust bean gum-poly (vinyl alcohol) interpenetrating polymer network microspheres for controlled oral drug delivery. J Drug Deliv Sci Technol 2016;33:1-2.

30. Reddy KM, Babu VR, Sairam M, Subha MC, Mallikarjuna NN, Kulkarni PV, et al. Development of chitosan-guar gum semiinterpenetrating polymer network microspheres for controlled release of cefadroxil. Des Monomers Polym 2006;9(5):491501.

31. Kaity S, Isaac J, Ghosh A. Interpenetrating polymer network of locust bean gum-poly (vinyl alcohol) for controlled release drug delivery. Carbohydr Polym 2013;94(1):456-67.

32. Bhattacharya SS, Shukla S, Banerjee S, Chowdhury P, Chakraborty P, Ghosh A. Tailored IPN hydrogel bead of sodium carboxymethyl cellulose and sodium carboxymethyl xanthan gum for controlled delivery of diclofenac sodium. Polym Plast Technol Eng 2013;52(8):795-805.

33. Menon TV, Sajeeth CI. Formulation and evaluation of sustained release sodium alginate microbeads of carvedilol. Int J Pharm Tech Res 2013;5(2):746-53.

34. Kulkarni RV, Sa B. Novel pH-sensitive interpenetrating network hydrogel beads of carboxymethylcellulose(polyacrylamide-grafted-alginate) for controlled release of ketoprofen: preparation and characterization. Curr Drug Deliv 2008;5(4):256-64. 